\title{
Miroirs, peignes et cosmétiques : objets de parure féminins dans les sanctuaires assyro-babyloniens
}

Mirrors, Combs and Cosmetics: Female Adornment in Assyro-Babylonian Sanctuaries

Laura Cousin

\section{OpenEdition}

\section{Journals}

Édition électronique

URL : https://journals.openedition.org/mondesanciens/3665

DOI : 10.4000/mondesanciens.3665

ISSN : 2107-0199

\section{Éditeur}

UMR 8210 Anthropologie et Histoire des Mondes Antiques

\section{Référence électronique}

Laura Cousin, « Miroirs, peignes et cosmétiques : objets de parure féminins dans les sanctuaires assyro-babyloniens », Cahiers « Mondes anciens » [En ligne], 15 | 2022, mis en ligne le 12 janvier 2022, consulté le 21 janvier 2022. URL : http://journals.openedition.org/mondesanciens/3665 ; DOI : https:// doi.org/10.4000/mondesanciens.3665

Ce document a été généré automatiquement le 21 janvier 2022.

\section{(c) (i) (9)}

Les Cahiers «Mondes Anciens » sont mis à disposition selon les termes de la licence Creative Commons Attribution - Pas d'Utilisation Commerciale - Pas de Modification 4.0 International. 


\title{
Miroirs, peignes et cosmétiques : objets de parure féminins dans les sanctuaires assyro-babyloniens
}

\author{
Mirrors, Combs and Cosmetics: Female Adornment in Assyro-Babylonian
}

Sanctuaries

Laura Cousin

\section{Introduction}

1 En Mésopotamie, les statues sont considérées comme de véritables incarnations des divinités. Faites d'une âme de bois, ou réalisées en pierre et recouvertes de métaux précieux, elles sont habitées par l'esprit divin et assurent la présence réelle du dieu ou de la déesse sur terre. Il convient donc d'organiser la vie de ces objets, de les loger dans des demeures dignes d'eux, de les nourrir, les vêtir, voire les distraire. Les statues prennent vie lors d'une cérémonie de consécration qui a lieu en deux temps, avec le "lavage de la bouche », mis pî, puis «l'ouverture de la bouche ", pit pî, pour que l'esprit du dieu entre dans la statue.

2 Les temples mésopotamiens abritaient des trésors, c'est-à-dire une accumulation de richesses sous la forme d'objets de culte (vaisselle sacrée en or ou en argent) ou de bijoux placés sur les statues. Ils se seraient multipliés pendant la seconde partie du premier millénaire av. J.-C., quand les temples étaient utilisés par les particuliers afin d'y déposer leur argent (Joannès 2005, p. 36). Les métaux précieux étaient alors entreposés dans des pièces de fonction que le sanctuaire mettait à leur disposition. Ces espaces étaient en liaison avec les activités exercées par le temple, mais les objets entreposés jouissaient d'un statut de bien privé. Ces réserves étaient alors appelées šutummu ou bìt qâtê. De plus, le temple possédait de l'argent et des biens pour son propre fonctionnement et pour mener ses activités économiques. Ainsi Dominique Charpin (2017, p. 64-69) compare les temples à des banques: c'est particulièrement le cas pour les sanctuaires dédiés au dieu Soleil Šamaš, et ce au moins 
depuis la période paléo-babylonienne (première moitié du deuxième millénaire av. J.C.). La documentation présente, en effet, un certain nombre de cas de prêts en or et en argent concédés par le dieu Šamaš.

Durant leur histoire, les temples babyloniens ont produit des inventaires de mobilier et de vaisselle sacrés, destinés à l'usage des dieux et des déesses qu'ils abritent. Pendant la période néo-babylonienne ( $\mathrm{vI}^{\mathrm{e}}$ siècle av. J.-C.), ce type de textes se présente sous la forme de bordereaux de contrôle sur lesquels sont notées les entrées et sorties des objets précieux appartenant au trésor divin. Parmi ces objets, sont parfois mentionnés des miroirs, des peignes et des pots à cosmétique, souvent évoqués dans la même partie du document. De plus, les inventaires exhaustifs décrivant le mobilier et la vaisselle des dieux sont fréquents en Mésopotamie. Ils commencent souvent par le terme šutukku signifiant « joaillerie », et permettaient d'exercer un contrôle étroit sur la circulation des objets précieux. Ces derniers pouvaient faire l'objet de vol, et le nombre d'objets précieux devait par ailleurs être soigneusement consigné, puisqu'il augmentait au gré des donations du roi et des particuliers (Beaulieu 1999, p. 141).

\section{Les inventaires référençant des parures divines}

Durant l'histoire de la Mésopotamie, on connait plusieurs grands inventaires : celui d'Ištar de Lagaba pour l'époque paléo-babylonienne, les inventaires de Qatna, pour l'époque médio-babylonienne (dans la deuxième moitié du deuxième millénaire av. J.C.) et enfin les inventaires des sanctuaires de l'Ebabbar, "Temple Brillant », de Sippar et de l'Eanna, «Temple du Ciel », d'Uruk pour l'époque néo-babylonienne ( $\mathrm{vi}^{\mathrm{e}}$ siècle av. J.-C.).

\section{L'inventaire d'Ištar de Lagaba (étudié par Leemans 1952)}

5 La tablette LB 1090, conservée à Leyde, présente une énumération d'habits et d'ornements destinés à la déesse Ištar (divinité des passions amoureuse et guerrière) de la ville de Lagaba, petite localité située entre Sippar et Babylone (d'après Jacquet à paraître). Il est ainsi question d'un certain nombre de bijoux en or, en pierres précieuses, en ivoire, ainsi que divers vêtements et sûrement de la vaisselle, comme des coupes en bronze, tous destinés à la déesse et à son culte.

\section{Les inventaires de Qatna (étudiés par Bottéro 1949, Fales 2004 et Muller 2015)}

6 C'est lors de sa deuxième campagne de fouilles à Qatna (Tell el-Mishrifeh, en Syrie actuelle) que le comte Robert du Mesnil du Buisson mit au jour dans la zone de la «Butte de l'Église » dix tablettes et fragments inscrits en cunéiforme, dans le bâtiment que les fouilleurs considéraient comme étant le sanctuaire de la déesse Nin-egal et qui est maintenant identifié comme le palais royal de Qatna. Il s'agissait en fait de quatre inventaires d'objets précieux, appartenant manifestement à Nin-egal, ainsi qu'aux "dieux du roi» (Bottéro 1949, p.9). Le culte de Nin-egal, la «Dame du palais », a commencé à prendre de l'importance en Mésopotamie à partir de la troisième dynastie d'Ur au XXI siècle av. J.-C. Il s'est ensuite sûrement propagé en Syrie au début du deuxième millénaire av. J.-C. Dans les deux premiers inventaires, le texte est divisé en 
six colonnes, tandis que les inventaires 3 et 4 sont inscrits sur des tablettes plus petites et comprennent une liste de bijoux décrits avec grande précision. Ces inventaires dénombrent en tout plus de 2000 objets, en majeure partie des bijoux et des vases. Dans ces inventaires, on trouve certes des bijoux, mais aussi une arme d'apparat, des habits, des vases précieux appartenant au nécessaire de toilette ou à la vaisselle de la divinité. Dans le premier inventaire, il est question d'un collier, constitué de pendentifs, dont l'un semble avoir l'aspect d'un miroir-nāmaru (1.313).

\section{Le trésor de l'Ebabbar de Sippar}

7 Francis Joannès (1992) a mis en lumière un dossier de textes listant le trésor du temple de Sippar, l'Ebabbar, et surtout les bijoux attribués aux déesses. Ces documents ne mentionnent pas de miroirs, peignes ou pots à cosmétique, toutefois comme les textes composant ce dossier présentent un aspect fragmentaire, cela n'exclut pas que des objets relatifs à la toilette aient pu s'y trouver. D'après les documents émanant du trésor de l'Eanna, Francis Joannès a proposé une reconstitution de l'aspect des bijoux. Ces derniers sont la propriété du dieu Soleil Šamaš, de sa parèdre Aya et de la déesse de la médecine Gula. En revanche, aucun trésor n'a pour l'instant été mis au jour dans l'Ebabbar de Larsa (l'autre sanctuaire majeur du dieu Soleil, situé dans le Sud de la Babylonie). Lors de la septième campagne de fouilles sur le site de Larsa en 1976, une jarre, vraisemblablement datée du règne de Samsu-iluna (1750-1711), a été découverte contenant de nombreux morceaux d'argent, quelques morceaux d'or, ainsi que des séries entières de poids en pierre et des outils en bronze. Les archéologues en ont d'abord déduit, au vu des éléments exhumés, qu'il pouvait s'agir du dépôt d'un orfèvre, nommé Ilšu-ibnišu (Arnaud et al. 1979). Ce dernier aurait alors placé dans une jarre ses instruments et les matériaux destinés à son travail et l'aurait enterrée dans le sol de son atelier. Cependant cette première interprétation a été remise en question (Bjorkman 1993), et il faut désormais plutôt y voir les instruments de travail d'un peseur (Charpin 2017, p. 86-99). Dix-huit scellements en argile ont, en effet, été retrouvés. Ils servaient à clore de petits sacs en textile ou en cuir, ensuite fermés par une cordelette. Les scellements portent les empreintes du sceau d'un personnage nommé Sin-uselli, fils d'Ilšu-bani, « peseur (kù-lá) de l'Egina d'Ur et serviteur de Samsuiluna ». L'Egina, temple de la déesse Kittum, devait à la fois servir à vérifier l'argent et aussi veiller à l'équité des transactions en argent ou en grain. Par conséquent, la jarre de Larsa et son contenu n'entrent plus dans l'étude des bijoux et parures.

\section{L'inventaire de l'Eanna d'Uruk}

Le texte NBC 4894, conservé dans la Yale Babylonian Collection aux États-Unis, est considéré comme l'un des inventaires les plus complets d'un trésor d'un sanctuaire babylonien, ici celui de l'Eanna d'Uruk («Temple du Ciel», dédié à la déesse Ištar) à l'époque néo-babylonienne. Il a été bien étudié par Paul-Alain Beaulieu en 1999, puis dans son ouvrage sur le panthéon d'Uruk (2003). Ce document est d'une nature particulière : l'administration du temple pouvait décider de réaliser un inventaire plus complet, si le nombre d'objets faisant partie du trésor divin augmentait, en raison, par exemple, de donations de rois ou de particuliers. Le document NBC 4894 est, par conséquent, un inventaire exhaustif sur 80 lignes d'objets appartenant aux déesses Ištar et Nanaya. On trouve des mentions de 52 colliers, de centaines de bijoux, d'autres 
objets précieux comme des perles, des figurines et des objets de toilette. Les lignes 74 à 76 concernent ces objets, parmi lesquels des peignes, des miroirs et des récipients à cosmétique, appelés mušālu ou muttabiltu.

Cette contribution cherche à déterminer, d'une part, si les miroirs, les peignes et les récipients à cosmétique, mentionnés dans la documentation de la pratique des temples, font partie de la vaisselle et de la joaillerie exclusivement attribuées aux déesses, et, d'autre part, dans quelle mesure ils sont associés plus largement au féminin dans la société mésopotamienne. Ainsi dans un premier temps, nous étudierons les miroirs comme parures destinées aux déesses dans le Proche-Orient ancien, puis dans un deuxième temps, nous ferons le point sur les peignes donnés aux déesses. Nous nous intéresserons, dans un troisième temps, à la préparation et à l'utilisation des cosmétiques dans les sanctuaires, avant de terminer sur l'association des miroirs, peignes et récipients à cosmétique dans certains documents textuels.

\section{Les miroirs comme parures destinées aux déesses dans le Proche-Orient ancien}

10 Les miroirs au Proche-Orient ancien sont connus grâce aux découvertes archéologiques, aux représentations iconographiques et à la documentation textuelle. S'ils font partie des objets attribués aux divinités, ils devaient aussi relever de l'ameublement des maisons des notables. Des miroirs ont, en outre, été retrouvés dans des tombes, montrant qu'ils devaient jouer un rôle apotropaïque pour le voyage des hommes dans l'au-delà.

\section{Présentation générale des miroirs}

Les premiers objets identifiés comme des miroirs ont été découverts sur le site néolithique de Çatal Hüyük dans le sud-ouest de l'Anatolie. Ils apparaissent en contexte privé et ont été mis au jour dans des tombes féminines datées des années 6000-5900 av. J.-C., au côté de bijoux, de paniers, de cuillères en os, de broches et de spatules. Les miroirs de Çatal Hüyük présentent une forme circulaire et un diamètre d'environ $9 \mathrm{~cm}$. Certains sont réalisés en obsidienne polie, et sont ensuite enduits à la chaux (Albenda 1985, p. 2).

De tels objets ont également été dégagés en Mésopotamie (pour un récapitulatif complet, voir Westenholz 2010, p. 466-469), par exemple à Uruk dans les niveaux du quatrième et $d u$ début du troisième millénaires av. J.-C. (miroirs en métal). À Tello, dans des niveaux datés des années 3200 av. J.-C., des tombes contenaient des pots de parfum, ainsi que de petits disques de cuivre qui seraient peut-être des miroirs. On trouve une situation similaire dans la ville de Kiš, près de Babylone, où des miroirs ont été exhumés au côté de bijoux, mortiers et pilons - peut-être pour fabriquer des cosmétiques - dans des tombes certainement féminines. Enfin, à Ur, des miroirs ont été dégagés dans des tombes datées de $2900 \mathrm{av}$. J.-C.

Ces miroirs se présentent souvent sous la forme de petits disques de bronze ou de cuivre, polis. Ils sont munis d'une tige qui s'insère dans une poignée verticale. Pour le deuxième millénaire av. J.-C., on a mis au jour des miroirs en bronze de forme similaire sur le site de Mari en Syrie dans des tombes féminines (Albenda 1985, p. 3 ; voir 
également Arkhipov 2012, p. 170-171 sur les miroirs dans les archives royales de Mari). Enfin, la découverte des tombes des reines de Nimrud en 1989 a permis d'exhumer des objets précieux, dont des miroirs en bronze et en électrum (Hussein 2016, p. 16).

Fig. 1 : Miroirs de la tombe II du site de Nimrud.

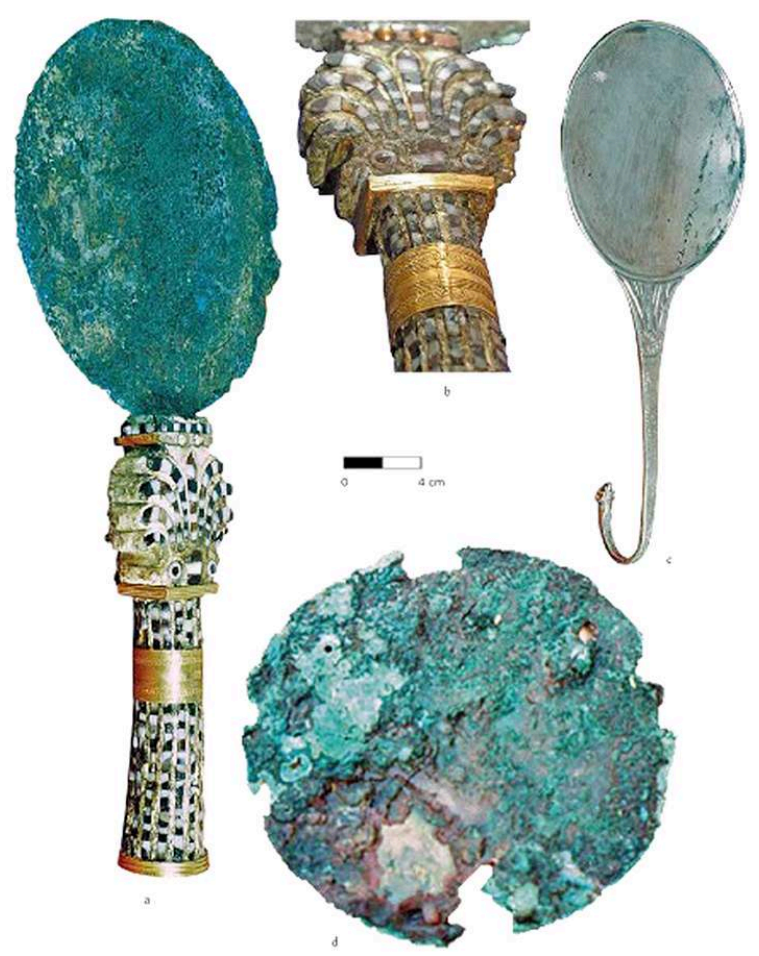

a et b : ND 1989.195 miroir en bronze avec détail de la poignée incrustée en forme de paume de la main ; c : ND 1989.194 miroir en électrum avec une inscription au nom d'Ataliya ; d : ND 1989.199 miroir en bronze.

D’après Hussein 2016, pl. 43.

Par ailleurs, les miroirs pouvaient être recouverts de cuir, comme le montrerait l'inventaire, très fragmentaire, d'époque kassite, IM 50083+50087, mis au jour sur le site de Dūr-Kurigalzu, non loin de Babylone et conservé au musée national d'Irak de Bagdad :

- 1 ki.min kuš.du ${ }_{8}$. ši.a na-ma-ru: «1 ditto (sicle ?) de cuir-dušûu (pour ?) un miroir?" (Gurney 1953, SUMER 9, texte $\mathrm{n}^{\circ} 25$, col. iii, l. 9).

- 7 ki.min kuš.sa ${ }_{5}$ na-ma-ru kuš.du ${ }_{8}$.ši.a x x : 7 ditto (sicle?) de cuir (pour?) un miroir?

(et du) cuir-dušû (Gurney 1953, SUMER 9, texte no 25, col. iv, l. 19 ; col. v, 1. 8, 13, 24).

Ce texte provient du palais royal de Dūr-Kurigalzu, et fait partie d'un lot plus conséquent de 29 tablettes étudiées par Oliver R. Gurney (1953). À l'exception de deux documents, les textes consistent en des bordereaux relatifs à des sorties d'or, d'argent et de pierres précieuses, utilisés pour des raisons variées. Il est également probable que ces matériaux précieux soient employés pour embellir les édifices royaux de la ville de Dūr-Kurigalzu. Ce passage de l'inventaire met en lumière une technique particulière appliquée au cuir, coloré grâce au cuivre utilisé comme un vert-de-gris (Steinkeller 2012, p. 264). Il est cependant à noter qu'il existe un autre terme nāmaru qui renverrait à un vêtement ou à un tissu (Durand 2009, p. 73); toutefois il est difficile de déterminer 
si l'inventaire IM 50083+50087 évoque plutôt un miroir recouvert de cuir ou un tissu agrémenté de cette matière.

D'autre part, un très rare miroir, sûrement originaire du site d'Isin, fait partie de la Yale Babylonian Collection (YBC 2453, voir Westenholz 2010) et porte l'inscription «Abzu, artisan du cuir » (ab-zu ašgab). Il date du III millénaire av. J.-C., et appartenait vraisemblablement à un homme de la ville d'Isin, située dans le Sud babylonien. Le fait que le nom d'un artisan du cuir soit inscrit sur un objet en métal interroge: cela signifie-t-il que le miroir lui appartenait? Ou bien a-t-il contribué à le fabriquer en réalisant sa couverture en cuir, si l'on admet que les miroirs pouvaient être recouverts de cuir?

On doit enfin une étude sur les miroirs de la période sassanide (224-651) à St John Simpson (2018) qui répertorie trois types de miroirs utilisés en Mésopotamie et en Iran à cette époque. Le premier est le miroir traditionnel, composé d'un disque circulaire en métal poli et doté d'une tige intégrée, s'insérant dans une poignée en bois ou en os. Le deuxième type était également constitué de métal poli et se présentait sous la forme d'un disque circulaire, souvent à face légèrement convexe, avec une poignée horizontale soudée à l'arrière. Ce type de miroir a été largement diffusé dans le monde méditerranéen et même en Asie. On trouve ensuite des miroirs dits « de poche » faits d'une fine feuille de verre circulaire convexe sertie dans un support solide. Certains miroirs plus communs étaient réalisés avec une monture en plâtre circulaire ou à pétales ; ces derniers ont été retrouvés en Syrie, à Palmyre et à Alep.

Le terme " miroir » est rendu de plusieurs façons par les dictionnaires, mais il convient cependant de reprendre les données dont nous disposons. Plusieurs termes, mušālu $(m)$ et nāmaru $(m)$ en akkadien, ainsi que (urudu)níg.šu.zabar et zabar.šu en sumérien, pourraient désigner les miroirs. Toutefois, d'après des recherches plus récentes, seul nāmaru $(m)$ désignerait avec certitude un miroir. Il vient du verbe amāru, "voir", renvoyant à la fonction même du miroir. La séquence (urudu) níg.šu.zabar est traduite par « instrument en bronze dans la main», parfois précédée du déterminatif "cuivre». Néanmoins, selon Joan Goodnick Westenholz (2010, p. 479-480), les objets connus sous les expressions ${ }^{(\text {urudu) }}$ níg.šu.zabar, voire zabar.šu, ne désigneraient pas un miroir, mais plutôt de la vaisselle fabriquée en matériaux précieux. Les dictionnaires évoquent par ailleurs le terme mušālu $(m)$ ou mašālu $(m)$, et qui viendrait lui-même du verbe mašălum, "être identique", comme le reflet d'une personne dans un miroir. Cependant, plusieurs chercheurs (Beaulieu 1999, p. 151 et Westenholz 2010, p. 480-481) s'accordent pour considérer que le mušālum désignerait plutôt un pot à cosmétique.

\section{Le miroir : objet féminin au premier millénaire av. J.-C.}

19 Le miroir constitue un motif iconographique ancien, qui s'est ensuite diffusé au premier millénaire av. J.-C. où il devient récurrent, représentant aussi bien des femmes appartenant à la royauté que des déesses, figurées assises ou debout et tenant un miroir (sur les déesses en général, voir l'ouvrage d'Asher-Greve et Westenholz 2013). La question se pose de l'intégration d'un tel motif dans l'art assyrien au I ${ }^{\mathrm{er}}$ millénaire av. J.-C.: il est très probable qu'il soit d'origine syrienne, puis ait été repris dans l'art impérial, bien qu'en Assyrie, ce soient plutôt des reines, mortelles, qui sont porteuses de miroir (Ornan 2002, p 473-474). 
20 Des images de femmes tenant des miroirs sont utilisées sur des sceaux dès l'époque médio-assyrienne (Ornan 2002, p. 464, fig. 7), mais la représentation la plus fameuse d'une femme de l'élite tenant un miroir est celle de Naqi'a-Zakûtu, mère du roi Assarhaddon qui a régné sur l'Assyrie entre 680 et 669 av. J.-C. Sur une plaque en bronze conservée au musée du Louvre (AO 20185) ${ }^{1}$, elle est figurée de profil tenant un miroir dans la main gauche et vraisemblablement une plante dans la main droite. Elle est, de plus, coiffée d'une couronne murale. Le contexte archéologique de ce document est perdu, mais il devait faire partie du socle d'un autel, ou éventuellement du dais d'un trône divin. La plaque pouvait plus précisément se trouver dans le temple de l'Ešarra ( Temple de l'Univers »), dédié au dieu Aššur et situé dans la ville d'Assur. De plus, le texte inscrit sur le relief décrit un rituel de naissance de statue divine mis pî. Naqi'a et le roi sont, ainsi, sûrement en train de se diriger vers la divinité en question.

21 Saana Svärd $(2015$, p. 77-80) a observé que, sur le bas-relief du musée du Louvre représentant Naqi'a et très vraisemblablement son fils Assarhaddon, la posture de la reine mère imite celle du roi, et par conséquent que les deux représentations peuvent être mises en parallèle. Ils portent tous deux quelque chose à leur visage avec leur main droite. Tandis que Naqi'a tient dans sa main gauche un miroir (symbole du féminin), le personnage masculin porte une masse (symbole de virilité). Quant à la couronne murale portée par Naqi'a, elle représente les remparts d'une ville et inclurait en son milieu un cône, afin d'imiter la tiare du roi. Cet ornement pourrait trouver son origine dans le couvre-chef des déesses et reines levantines (Svärd 2015, p. 79-80).

Un autre bas-relief assyrien, conservé au Vorderasiatisches Museum de Berlin et représentant sûrement Liballi-šarrat, la reine d'Assurbanipal (669-631), comporte de nombreuses similitudes avec la plaque en bronze sur laquelle figure Naqi'a. Sur cette stèle fragmentaire (fig. 2), le personnage féminin se tient dans la même position que la reine mère Naqi'a, et porte aussi une couronne murale. Malgré l'état fragmentaire de l'objet, on peut raisonnablement proposer qu'elle tienne un miroir dans sa main gauche et une plante dans sa main droite qu'elle porte à son visage (voir Brereton 2018, p. 17). 
Fig. 2 : Stèle fragmentaire représentant la reine Liballi-šarrat.

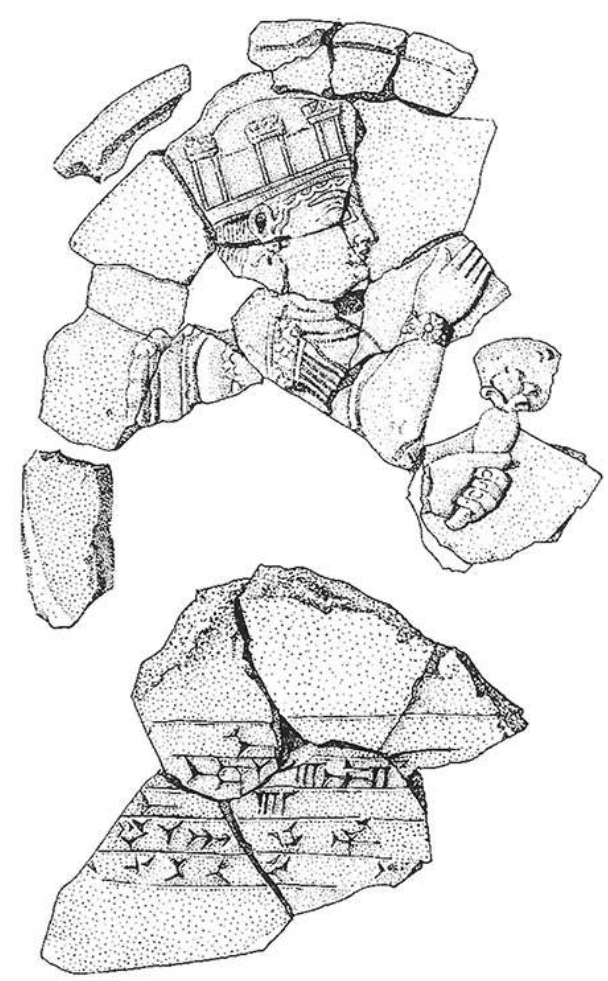

Originaire de la ville d'Assur. Berlin, Vorderasiatisches Museum, VA 8847.

D'après Alvarez-Mon 2009, pl. 13.

Enfin, et même si cette question ne peut être élucidée pour l'instant, on peut se demander plus généralement quelle est la signification du symbole du miroir, ce dernier pouvant peut-être être lié à la beauté, à la brillance voire à une certaine forme de pouvoir féminin.

\section{Miroirs et divinités féminines}

Parmi les déesses proche-orientales les plus représentées tenant un miroir, on compte la divinité anatolienne Kubaba, parèdre du dieu de l'orage et déesse de la fertilité, figurée sur les stèles de Malatya et Karkemiš, conservées à Istanbul et datées du $\mathrm{IX}^{\mathrm{e}}$ siècle av. J.-C. (Rova 2008, p. 561). Kubaba est présentée assise sur un trône, lui-même reposant sur un lion couché. Cela n'est pas sans rappeler certaines scènes du bol en or de Hasanlu, découvert en 1957 dans le nord-ouest de l'Iran, et daté entre 1200 et 900 av. J.-C. L'une d'entre elles montre une déesse debout contre un lion rugissant, qui tient dans sa main gauche un miroir et dans sa main droite une masse d'arme. Selon MarieThérèse Barrelet (1984, p.134), cette divinité pourrait résulter de plusieurs syncrétismes, notamment entre l'šstar mésopotamienne, qui a le lion pour symbole, et l'Astarté phénicienne qui porte une massue et un miroir.

Il est à noter que dans la documentation textuelle hittite des XIV et XIII siècles av. J.-C., le miroir est également essentiellement associé aux déesses (Hoffner 1966, p. 331-333), de même que dans l'iconographie de Nuzi au XIve siècle av. J.-C. (Ornan 2002, p. 472, fig. 16). 
plusieurs déesses: Kubaba en Anatolie, puis Gula Ištar, Nanaya et Ningal en Mésopotamie. Concernant Gula, la déesse de la médecine, le texte SAA 20, 30, d'époque néo-assyrienne (911-610 av. J.-C.) et issu d'archives de particuliers, présente un rituel et des libations offertes à la déesse. Parmi les éléments utilisés dans ce rituel, on trouve un miroir (1. 12-17) :

[Les officiants] ... et se tiennent devant Gula. Le prêtre ouvre la porte, place [un s]iège dans la cour, [et met] (un collier) de perles de cristal et un miroir en argent sur elle. Devant elle, il étend un [...]. Il prépa[re] des tranches de pain huilées dans un panier, met un [mo]uton grillé dessus, et les met à table. [Il off]re du sel devant [ND], fait une offrande de farine et réalise des libations. Le maître du banquet [...]. [Après qu'il] a placé [...] avec sa main, tu placeras le collier de perles de cristal autour de son cou. Elle portera le mir[oir] [d'argent...] dans sa main gauche.

Il est, par conséquent, intéressant de remarquer qu'après avoir été parée d'un collier Gula doit porter le miroir dans sa main gauche, à l'instar de Naqi'a et de la reine Liballišarrat.

Pour Ningal, la parèdre du dieu Lune Sîn, déesse associée à la fertilité honorée dans la ville d'Ur, la lettre $A B L 1246$ (1. 8) la met en rapport avec un miroir :

Je prendrai la table, la figurine en or d'un taureau sauvage et le miroir en or et les donnerai à Ningal.

Ce rituel rappelle une cérémonie égyptienne, durant laquelle une prêtresse offre un miroir en offrande à une déesse. La déesse y voit sa beauté se refléter et s'en réjouit (Husson 1977, p. 23 et 269-274). Cependant, au cours de l'histoire de la Mésopotamie, le miroir ne semble pas avoir été toujours exclusivement associé à une divinité féminine. L'inventaire du dieu de l'orage Adad de la ville Me-Turan, localité située dans la vallée de la Diyala, montre qu'il reçoit deux miroirs (col. i, l. 2' et col. ii, 1.11'). Le texte est daté du règne de Șilli-Sîn d'Ešnunna (1764-1762) : « $\left[\mathrm{x}^{+}\right] 2$ nāmarum mi ni x x kù.babbar ", « $[\mathrm{x}] 2$ miroirs ... argent» (Black et Al-Rawi 1983). Dans ce document très fragmentaire, seule la dernière ligne (col. iv, l. 3) précise que ces objets appartiennent «à Adad ». On peut ainsi se demander si la parèdre d'Adad, Šala, n'apparaîtrait pas également dans cet inventaire, pour se faire remettre les miroirs.

Enfin, le miroir pourrait être plus qu'une parure, peut-être un emblème divin comme le montre la lettre SAA 1, 77 (1. 1-9), dans laquelle on trouve la mention suivante :

$\mathrm{Au}$ roi (Sargon II), mon seigneur, ton serviteur Țab-șilli-Ešarra. Salut au roi, mon seigneur ! Puissent (les dieux) Aššur et Mullissu bénir le roi, mon seigneur ! La paire de miroirs ( ${ }^{\mathrm{d}}$ nam-ra-ni) en bronze est très belle. Ils sont en train de travailler sur leurs finitions.

31 Cette missive est suivie de la lettre SAA 1, 78, dans laquelle le même homme Țab-șilliEšarra évoque à nouveau le sujet des miroirs (1. 6-8) :

[Nous] avons ôté le miroir ( ${ }^{\mathrm{d}}$ nam-ru) le $1^{\mathrm{er}}$ [jour] et nous l'avons placé devant son visage.

Les éditeurs du recueil SAA 1 ont initialement proposé de traduire namru et namrani par "panthère ", tout en les laissant en italique, afin de montrer que cette lecture restait incertaine. Cependant, le Chicago Assyrian Dictionary (CAD N/1, p. 219a) précise bien que le miroir nāmaru peut s'écrire namru et que son pluriel peut donner namrāni. Si les lettres SAA 1, 77 et 78 ne donnent aucun indice quant à l'emplacement de ces miroirs, il est surtout intéressant de constater que les termes namru et namrani sont précédés du déterminatif accompagnant les noms de dieux (dingir), et que les objets devaient par 
conséquent être placés dans un sanctuaire. On pourrait y déceler un indice montrant que le miroir serait lui-même devenu une manifestation de la divinité.

\section{Les peignes donnés aux déesses}

Le peigne, mušț ou mulțu en akkadien, constitue un autre élément de l'inventaire des objets offerts aux déesses Ištar et Nanaya de l'Eanna d'Uruk. Un passage célèbre du poème Dumuzi et Inanna (le nom sumérien de la déesse Ištar), daté de la troisième dynastie d'Ur (2112-2004 av. J.-C.) raconte les différentes étapes de la préparation de la déesse, qui s'apprête à retrouver son amant Dumuzi, par lesquelles un soin particulier est apporté à ses cheveux :

Elle mélange? du khôl. Elle laisse tomber ses cheveux qui ont été peignés. Elle se lave et se frotte avec du savon. Elle se frotte avec le savon du bol blanc, elle se lave avec l'eau de l'aiguière sacrée. Elle s'oint d'huile douce du bol de pierre. Elle s'habille dans ses vêtements lavés. Elle se peigne les cheveux qui ont été défaits. Elle peint ses yeux avec du khôl. Elle met les perles de lapis-lazuli sur son cou, en arrangeant leur bouton sur les tendons de son $\mathrm{cou}^{2}$.

Cependant, le peigne est un élément qui apparaît ponctuellement comme objet attribué à d'autres déesses. En outre, une empreinte de sceau-cylindre d'époque médioassyrienne figure un personnage féminin (reine ou déesse) tenant un miroir et se faisant coiffer avec un peigne. On semble par ailleurs lui présenter un bol, peut-être un pot à onguent (Ornan 2002, p. 464, fig. 7).

\section{Le peigne donné à Hušbišag}

Dans le texte daté de la troisième dynastie d'Ur (2112-2004 av. J.-C.), appelé La mort d'UrNammu et sa descente aux Enfers (Kramer 1967), le roi Ur-Nammu (2112-2095) dit avoir offert à la déesse Hušbišag - parèdre d'un dieu mineur du monde souterrain, Namtar un coffre spécial, une broche pour les cheveux et un, voire plusieurs, peigne(s) (1. 109-112) :

À Hušbišag, l'épouse de Namtar, dans son palais, le berger Ur-Namma a offert un coffre? avec un manche en lapis-lazuli, contenant ? tout ce qui est essentiel dans le monde souterrain, un fermoir en argent orné de lapis-lazuli, et un peigne de mode féminine.

Cependant, d'autres traductions proposent qu'Ur-Nammu n'ait pas offert un mais sept peignes à la déesse ${ }^{3}$.

\section{Le peigne de Ningal}

37 Après une expédition à Dilmun, la déesse Ningal a reçu dans son temple à Ur un peigne en ivoire (1 ga-ríg zú-am-si dans UET 5 678), durant l'époque paléo-babylonienne, dans la première moitié du deuxième millénaire (Maggio 2012, p. 90).

\section{Le peigne dans le rituel contre la Lamaštu}

38 Il s'avère, enfin, que les peignes étaient utilisés dans certains rituels magiques, notamment contre la démone Lamaštu : 
C'est le rituel: Tu réalises une fille d'Anu (= Lamaštu) en argile. Tu lui couvres la tête avec des cheveux. Tu lui donnes pour vêtement une serviette souillée. Tu la piques avec une épine de palmier-dattier. Tu lui donnes un peigne, une quenouille et une petite flasque d'huile ${ }^{4}$. Tu remplis ses sacs en cuir de gruau, de malt, d'une préparation brassée, d'orge grillé, de pain séché ; tu lui fais quatre ânes en argile. $\mathrm{Tu}$ lui donnes ces choses comme provisions de voyage (Rituel 3: Farber 2014, p. 151).

On trouve une variation au rituel accompli contre la Lamaštu (Farber 2014, p. 187), avec cette fois le don d'un peigne et d'un miroir à la démone :

Quand tu veux faire le rituel contre la Lamaštu, tu dessines une fille d'Anu sur le côté droit de la porte, sur le côté gauche, ou en direction de l'entrée de la chambre. À la droite et à la gauche de la fille d'Anu (tu mets) un chien, un cochon, une lampe, un scorpion, une quenouille, un peigne, un miroir et une cheville d'âne. Elle doit tenir des serpents dans ses mains.

Bien qu'appartenant au monde des démons, la Lamaštu est considérée comme une déesse par les Mésopotamiens. Elle est la fille du dieu du ciel Anu et l'une des sœurs d'šstar. Elle fut cependant expulsée des cieux pour avoir mangé un nourrisson. De fait, on considère qu'elle s'attaque surtout aux femmes enceintes, aux jeunes mères et à leurs bébés. Elle symbolise, entre autres, la fièvre puerpérale, qui apparaît lorsque le placenta n'a pas été complètement expulsé, ou encore la mort subite du nourrisson. Pour l'éloigner, il faut lui offrir un certain nombre d'objets. Ainsi, pour qu'elle entreprenne son voyage à travers la steppe, elle doit disposer de provisions, mais aussi d'un âne, d'un bateau pour traverser les rivières et d'ustensiles a priori de mise en beauté féminins, comme un peigne ou un miroir.

\section{Les cosmétiques, leur préparation et leur utilisation dans les sanctuaires}

Les sanctuaires apparaissent comme les lieux de production d'un certain nombre de produits cosmétiques (huiles et eaux parfumées par exemple), et les inventaires des temples présentent, quant à eux, des objets appartenant à la vaisselle sacrée des dieux pouvant servir de contenants à ces produits (voir Casanova 2008 pour une étude des vases à parfum et boîtes à fard aux $\mathrm{IV}^{\mathrm{e}}-\mathrm{II}^{\mathrm{e}}$ millénaires).

\section{Les fards et onguents}

Parmi les cosmétiques, les dieux semblent peut-être recevoir des fards, mais nous disposons de peu de renseignements quant aux techniques utilisées et aux produits les composant. Dans le récit Dumuzi et Inanna précédemment cité, la déesse s'applique sur les yeux un produit appelé guhlu qui est un dérivé du kôhl, soit une pâte noire-bleutée destinée à farder les yeux et souligner l'intensité du regard (à propos du guhlu, voir Potts et al. 1996).

En outre, les fards peuvent parfois être présentés dans des coquillages. Ces derniers viennent vraisemblablement du golfe Persique ou de l'océan Indien. Parmi les coquillages, on trouve des variétés appelées en akkadien ayyartum (peut-être le cauri), kapașum et lahyanatum (Maggio 2012, p. 91-92). 
Trois textes paléo-babyloniens mentionnent des coquillages offerts au temple de la déesse Ningal :

- UET 5, 546 (règne de Gungunum, $\mathrm{xx}^{\mathrm{e}}$ siècle av. J.-C.) : " quatre sila et cinq sicles de coquillages-ayyartum; trois sila et dix sicles de coquillages- kapașum et de coquillages-lahyanatum ».

- Nisaba 19, 209 (règne d'Abi-sare début xIx siècle av. J.-C.) : «[x] sicles de coquillages-ayyartum ».

- UET 5, 678 (non daté) : " neuf sila de coquillages-ayyartum ».

Par ailleurs, d'après les découvertes archéologiques, l'utilisation de coquillages comme récipients est bien attestée dans le Proche-Orient, par exemple sur des sites paléolithiques en Israël, ou encore en Oman, en Arabie, mais également à Chypre ou en Crète (Prieur 2005, p. 165). De plus, des coquillages utilisés comme récipients à cosmétique ont été mis au jour en Oman, aux Émirats Arabes Unis et en Grèce (Prieur 2005, p. 165), mais aussi dans les tombes syro-mésopotamiennes à l'âge du Bronze. Ce sont des mollusques aux formes généralement creuses qui sont alors utilisés, certains pouvant même servir à recueillir de l'huile (Prieur 2005, p. 165).

D'autre part, le Penn Museum (University of Pennsylvania Museum of Archaeology and Anthropology) présente sur son site Web plusieurs coquillages servant de récipients à cosmétique, avec ce qu'il semble être des fards à l'intérieur ${ }^{5}$. Certains viennent plus précisément des fouilles d'Ur et du secteur des tombes royales du III ${ }^{\mathrm{e}}$ millénaire av. J.-C.

\section{Les pots à cosmétique}

Si la composition et l'usage des cosmétiques dans les sanctuaires sont difficiles à déterminer, un petit billet (NCBT 72 ; voir Nemet-Nejat 1993) concerne la réalisation d'un élément d'un pot à cosmétique, confié aux orfèvres du temple de l'Eanna d'Uruk : « Un couvercle d'un pot (mušălu) en or appartenant à la Dame d'Uruk, se trouve dans l'atelier des orfèvres. Fait le 9 d'Arahsamnu de l'an 3 de Nériglissar (558), roi de Babylone».

8 Ce pot est, en plus, doté d'une «bouche (ká)» que Paul-Alain Beaulieu (1999, p. 151) propose de traduire par goulot. Si le $C A D(M / 2$, p. 256-257) est peu clair quant à la signification du terme mušălu, indiquant qu'il pourrait s'agir à la fois d'un miroir et d'une palette à cosmétique, plusieurs spécialistes s'accordent pour considérer que mušālu serait bien un récipient à cosmétique. Déjà dans la documentation de Mari, datée de la première moitié du deuxième millénaire, Jean-Marie Durand avait mis en évidence un objet mušălu (ARM 21, 222, 1. 29) et avait proposé la traduction de «vase à onguent ». Cette traduction est ensuite reprise par Michaël Guichard (2005, p. 243). On doit ensuite à Walter Farber la traduction de pot à onguent (1987, p. 94-95), plutôt que miroir. D'autre part, le document NBC 4894 consacré à l'inventaire de joaillerie de l'Eanna d'Uruk distingue bien, d'une part, un miroir en or, et d'autre part, le pot à cosmétique en or :

74. [...]; un pot-mušălu en o[r] ;

75. un miroir en or ; deux oiseaux en or ; deux mumal[li x x] ;

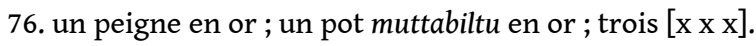

On connaît un autre pot-mušālu en or dans la documentation néo-babylonienne, mentionné dans le texte TCL $1239: 2$ (Moore 1975, p. 48-49), sans qu'il soit possible de déterminer s'il s'agit du même récipient : « $1 \frac{1}{3}[\ldots], 4 \frac{1}{2}$ sicles d'or, un pot en or, 2 [...], 81 poids en or, 50 cornalines, 50 lapis-[lazuli], 2 ornements pour des déesses. Reçus par 
Nadin, fils de Marduka, Nabû-šum-ukin [fils de] Nergal-uballit, orfèvres. Ils ont été amenés à Babylone avec les dieux. Fait le 24 de Tašritu de l'an 22 de Nabuchodonosor (583), roi de Babylone ».

D'autre part, le terme muttabiltu que l'on trouve dans NBC 4894, l'inventaire des objets sacrés destinés aux déesses d'Uruk, Ištar et Nanaya, désignerait aussi un pot en or. Paul-Alain Beaulieu (1999, p. 183-188) a mis en lumière un autre texte d'Uruk, PTS 3238, lequel mentionne un pot-muttabiltu, mais qui est qualifié de muttabiltu ša aban zakukû, désignant le verre, la pâte de verre, ou la fritte. Il s'agirait de fait plutôt d'un petit vase.

Le texte PTS 3238 est intéressant à plus d'un titre. D'une part, Kaššaya, l'une des filles de Nabuchodonosor II, roi de Babylone durant l'apogée de l'empire au vi ${ }^{e}$ siècle av. J.-C., fait une donation au temple d'Uruk, et plus précisément à la déesse Nanaya, sûrement lors de la $15^{\mathrm{e}}$ année de règne de son père (sur la princesse Kaššaya et ses sœurs, voir Beaulieu 1998). D'autre part, c'est un témoignage des plus remarquables concernant l'implantation de la famille de Nabuchodonosor II dans la ville d'Uruk, où il avait exercé une fonction administrative très élevée dans le temple de l'Eanna, avant la prise du pouvoir par son père, Nabopolassar (Jursa 2007) :

1.11 : [o] muttabiltu de verre

1. 12 : Total de l'apport de Kaššaya, fille du roi

1.13 : donné pour la besace-pišannu de Nanaya.

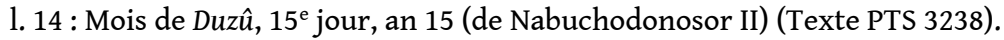

Les pots à cosmétique se retrouvent également dans certaines compositions de dot, comme le montre le texte paléo-babylonien UET 5, 793 (l. 6), découvert à Ur. Il liste la dot d'une jeune femme, appelée Rubatum, entrant dans la maison de son mari et qui apporte avec elle deux mu-ša-lu-um ud.ka.bar.ra, donc vraisemblablement deux pots à cosmétique en bronze.

\section{L'huile parfumée}

53 Les sanctuaires babyloniens produisaient des parfums, sous la forme d'huiles et plus rarement d'eaux parfumées (d'après Cousin à paraître). L'application d'huile est un élément indispensable à la toilette des hommes et des femmes, elle permet de redonner souplesse et brillance à la peau. L'éclat de la peau huilée est signe de prospérité et de bonne santé, et l'on trouve également un caractère mystique de la «bonne odeur » censée émaner des dieux et des rois. De fait, les brûle-parfums font partie du mobilier cultuel.

À l'époque paléo-babylonienne, une inscription commémorative fragmentaire conserve le texte d'une dédicace d'un temple par le roi Rim-Sîn de Larsa, appelé E-irara (é-ì-rárá), et qualifié de sanctuaire « dont le parfum est agréable et dont les bois aromatiques sont précieux ». Ce nom cérémoniel en sumérien donne en akkadien bit raqqîm, soit « parfumerie » (Charpin 2017, p. 185-186). Toujours dans le royaume de Larsa, un lot de 63 textes (fin $\mathrm{Xx}^{\mathrm{e}}$ - début $\mathrm{XIX} \mathrm{x}^{\mathrm{e}}$ siècle av. J.-C.) fait état de livraisons d'huiles parfumées comme rations pour des individus, pour le roi, ainsi que pour les divinités. L'huile pouvait alors servir à nettoyer les objets dans le palais ou les temples (Middeke-Conlin 2014).

Dans certains temples, on trouve aussi la présence de prébendes de parfumeurs, même si les attestations sont rares pour le moment. Cependant, dans le recueil récent des textes paléo-babyloniens de la collection Hilprecht de Iéna édité par Anne Goddeeris 
(2016, texte 13), il est question d'une prébende de parfumeur attachée au temple de l'Ekur à Nippur.

Dans la documentation néo-babylonienne, les temples fournissent les meilleurs exemples de fabrication du parfum (sur les parfumeuses au I ${ }^{\text {er }}$ millénaire av. J.-C., voir Cousin 2016). Dans l'archive du temple de l'Ebabbar de Sippar, "Temple brillant", étudiée par Herman Bongenaar (1997), différentes huiles parfumées apparaissent dans un lot de documents concernant le groupe de prébendiers formé par les presseurs d'huile du temple (Bongenaar 1997, p. 261-287). Les parfums peuvent aussi être brûlés et prennent alors la forme de fumigation, tandis que les huiles parfumées peuvent être utilisées pour enduire les statues cultuelles.

Ces huiles sont employées lors de moments spéciaux, voire de fêtes, et pour des déesses particulières. L'une d'elles est appelée hilșu, terme qui peut désigner une huile parfumée, aussi bien que la cérémonie pour laquelle cette huile est fabriquée. Une recette pour réaliser le hilṣu est connue grâce au texte BM 74485 : on aurait ainsi besoin de 14 aromates, pour un poids total de $9,4 \mathrm{~kg}$, mélangés à 42 litres d'huile de sésame. D'autre part, le hilșu semble uniquement associé à la déesse Šarrat-Sippar (la « Reine de Sippar »), et notamment à la cérémonie de son mariage sacré, comme l'indiquent les textes d'époque perse-achéménide Cyr.279 et Camb.152. Enfin, d'après la prosopographie des prébendiers du sanctuaire de Šarrat-Sippar, l'E-edin-na, la fabrication d'huile parfumée semble réservée à la famille de Sîn-ili, fils de Šamaš-iddin et descendant de Bel-êtẹeru, qui est l'un des èrib bīti «entrants » du temple de ŠarratSippar.

Dans l'archive du temple de l'Eanna d'Uruk, plusieurs documents mentionnent un bâtiment appelé bìt hilși, «maison où l'on filtre et raffine l'huile ». L'un d'entre eux, UCP $9 / 2,27$, conservé au Hearst Museum, est daté du règne de Nabuchodonosor II et a été étudié par Francis Joannès (2006, p. 76-77). Il détaille les ingrédients donnés à un homme appelé Nabû-mušetiq-udê en vue du «travail pour le bit hilși ». Il obtient ainsi 22 produits aromatiques différents (cèdre, cyprès, myrte, buis, roseau odorant, myrrhe par exemple), mais également des pois, de la laine et du sésame. Le bit hilși est placé sous la responsabilité d'un prêtre appelé šangû bìt hilși, et est associé aux déesses Ușuramâssu et Urka'itu (2006, p. 77-83). Un autre bit hilṣi est attesté dans la ville d'Ur, d'après une brique datée du règne du roi babylonien Nabonide (556-539), et est spécifiquement dédié à la déesse Ningal :

(Moi) Nabonide, roi du monde, roi de Babylone, qui a bâti pour Ningal, sa Dame, l'Enunmah, le bìt hilși à l'intérieur de l'Ekišnugal (UET 1, 189 ; voir Schaudig 2001, p. 339-340).

Enfin, à l'époque séleuco-parthe, et plus précisément à Babylone, on trouve un bit hilși dans le temple de l'Esabad, dédié à la déesse de la médecine Gula. Comme l'indique Francis Joannès (2006, p. 88) :

[...] ce peut être, aussi bien, une ultime spécialisation de cette officine, liée à des soins thérapeutiques qui auraient été dispensés dans le temple de Gula. 


\section{L'association entre les miroirs, les peignes et les boîtes à cosmétique}

\section{Dans les textes de l'époque d'El Amarna}

La mise en rapport entre les miroirs, les pots à cosmétique et le monde féminin trouve un écho avec la liste EA 25 (voir Moran 1987), découverte sur le site d'El Amarna, ancienne capitale du pharaon égyptien Akhenaton, et envoyée depuis le Mitanni. Ce long texte, daté du XIv ${ }^{\mathrm{e}}$ siècle av. J.-C., se compose de quatre colonnes d'environ 75 lignes chacune. Il s'agit de la dot que Tušratta, roi du Mitanni, a donnée, peut-être à l'occasion du mariage de sa fille avec le pharaon Aménophis III. Sur la deuxième colonne, entre les 1.43 à 52, il est question de plusieurs récipients à onguent. Puis, les 1. 56-59 mentionnent des miroirs :

Un miroir en argent, 40 sicles de poids, la poignée figurant une femme, en ivoire. Il a été plaqué de $1 \frac{3 / 4}{4}$ sicles d'argent. Un miroir en argent, 40 sicles de poids: la poignée figurant une femme en ébène. Il a été plaqué de $13 / 4$ sicles d'or.

61 Cette colonne se termine avec la mention de 100 peignes en argent. La colonne iii évoque, quant à elle, des miroirs avec leurs étuis (au moins des 1.16 à 23). Enfin, la col. iv mentionne plusieurs récipients à parfum (1. 51-55) :

Un récipient à parfum avec de l'huile parfumée à la myrrhe. Un récipient à parfum avec de l'huile-kanatku. Deux récipients à parfum avec de l'huile-sikil. Un récipient à parfum avec de l'huile de sureau. Deux récipients à parfum avec de l'huile de myrte. Un récipient à parfum avec de l'huile-peršantu. Un récipient à parfum avec de l'huile de sureau. Un récipient à parfum avec de l'huile de myrte. 10 pots-kirru qui sont pleins d'huile douce.

\section{Dans des rituels de Babylone}

Deux tablettes rituelles écrites en caractères cunéiformes et récemment éditées par Rocío Da Riva et Gianluca Galetti (2018) mettent en lumière les premiers jours du rituel de l'Akitu de Nisanu, c'est-à-dire de la fête du Nouvel An babylonien, qui a lieu durant douze jours au moment de l'équinoxe de printemps. Ces deux documents, datés des $\mathrm{VI}^{\mathrm{e}}$ $\mathrm{V}^{\mathrm{e}}$ siècles av. J.-C., comprennent des descriptions du rituel, notamment des libations, récitations, chants, purifications, dons d'objets, parmi lesquels des miroirs, peignes et objets cosmétiques. Ainsi lors du premier jour du mois de Nisanu (BM 40790, col. ii, 1. 10-12), une officiante du culte, appelée hullālānitu, prend dans une besace-pišannu des miroirs pour les déesses Nanaya, Sutìtu et Gula de l'Eulla (l'Eulla, « Maison de la Joie » est le temple de la déesse Gula à Sippar). Toujours dans BM 40790 (col. i, 1. 21-22), des chanteuses semblent offrir un peigne, évoqué sous le terme mumarritu en akkadien (sur ce mot, voir Da Riva et Galetti 2018, p. 207). Dans le texte BM 40854+BM 41208 (col. iv, 1. 1-11), la même officiante hullālānitu donne un miroir à Beltiya, la parèdre du dieu de Babylone, Marduk.

On constate par conséquent une pratique genrée de ces rituels : dans les cas décrits par les rituels de la ville de Babylone, il s'agit d'officiantes femmes qui prennent soin des déesses, avec certains objets manifestement associés à la féminité. 


\section{Conclusion} l'iconographie assyrienne, notamment par la reine Naqi'a, mère du souverain Assarhaddon, ils apparaissent dans les listes de dots de certaines princesses ou femmes de la notabilité, et sont associés de façon privilégiée aux déesses. Enfin, toujours dans le cas des miroirs, ces objets semblent parfois dépasser leur statut de parure en devenant de véritables emblèmes divinisés à l'époque néo-assyrienne, comme le montreraient les lettres SAA 1, 77 et 78. L'exemple des miroirs, des peignes et des cosmétiques montre le lien existant entre ces parures, l'acte de parer et le divin, ce qui se manifeste particulièrement dans les textes de rituels issus des sanctuaires de la ville de Babylone au premier millénaire av. J.-C.

\section{BIBLIOGRAPHIE}




\section{Abréviations}

$A B L$ : R. F. Harper, Assyrian and Babylonian Letters, Londres, 1892-1914.

AO : département des Antiquités orientales du musée du Louvre, Paris.

ARM : Archives Royales de Mari.

$\mathrm{BM}:$ British Museum, Londres.

CAD : I. J. Gelb et al. (dir.), The Assyrian Dictionary of the University of Chicago, Chicago, 1956-2010.

Camb. : J. N. Strassmaier, Inschriften von Cambyses, König von Babylon, Leipzig, 1890.

Cyr. : J. N. Strassmaier, Inschriften von Cyrus, König von Babylon, Leipzig, 1890.

EA : J. A. Knudtzon, Die El-Amarna-Tafeln, Leipzig, 1915.

IM : Iraq Museum, Bagdad.

LB : tablettes cunéiformes de la collection Liagre Bohl, Leyde.

NBC : Nies Babylonian Collection, Yale Babylonian Collection, New Haven.

NCBT : tablettes cunéiformes de la Newell Collection of Babylonian Tablets (Yale Babylonian

Collection, New Haven).

Nisaba 19 : J. A. Black et G. Spada, Texts from Ur kept in the Iraq Museum and in the British Museum, Messine, 2008.

PTS : tablettes cunéiformes du Princeton Theological Seminary.

SAA : S. Parpola et al. (éd.), State Archives of Assyria, Helsinki, 1987-2018.

TCL : textes cunéiformes du musée du Louvre.

UCP : University of California Publications in Semitic Philology.

UET : Ur Excavations Texts.

VA : Vorderasiatisches Museum, Berlin.

YBC : tablettes cunéiformes de la Yale Babylonian Collection (New Haven).

\section{Bibliographie générale}

Albenda P. (1985), « Mirrors in the Ancient Near East », Notes in the History of Art 4-2/3, p. 2-9.

Alvarez-Mon J. (2009), « Ashurbanipal's Feast: A View from Elam », Iranica Antiqua 44, p. 131-180.

Arkhipov I. (2012), Le vocabulaire de la métallurgie et la nomenclature des objets en métal dans les textes de Mari. Matériaux pour le Dictionnaire de Babylonien de Paris. Tome III, Archives Royales de Mari 32, Louvain-Paris-Walpole.

Arnaud D., Calvet Y. et Huot J.-L. (1979), « Ilšu-ibnišu, orfèvre de l'E.Babbar de Larsa. La jarre L. 76.77 et son contenu », Syria 56, p. 1-64.

Asher-Greve J. M. et Westenholz J. G. (2013), Goddesses in Context: On Divine Powers, Roles, Relationships and Gender in Mesopotamian Textual and Visual Sources, Orbis Biblicus et Orientalis 259, Fribourg-Göttingen.

Barrelet M.-Th. (1984), « Le décor du bol en or de Hasanlu et les interprétations proposées à son sujet » dans Barrelet M.-Th. éd., Problèmes concernant les Hurrites 2, Paris. 
Beaulieu P.-A. (1998), « Ba'u-asītu and Kaššaya, Daughters of Nebuchadnezzar II », Orientalia 67-2, p. $173-201$.

Beaulieu P.-A. (1999), « Un inventaire de joaillerie sacrée de l'Eanna d'Uruk », Revue d'Assyriologie et d'archéologie orientale 93, p. 141-155.

Beaulieu P.-A. (2003), The Pantheon of Uruk during the Neo-Babylonian Period, Cuneiform Monographs 23, Leyde-Boston.

Bjorkman J. K. (1993), «The Larsa Goldsmith's Hoards - New Interpretations », Journal of Near Eastern Studies 52, p. 1-23.

Black J. A. et Al-Rawi F.N.H. (1983), « The Jewels of Adad », SUMER 39, p. 137-143.

Bongenaar A.C.V.M. (1997), The Neo-Babylonian Ebabbar Temple at Sippar: Its Administration and Its Prosopography, Publications de l'Institut historique-archéologique néerlandais de Stamboul 80, Leyde.

Bottéro J. (1949), «Les inventaires de Qatna », Revue d'Assyriologie et d'archéologie orientale 43, p. $1-40$.

Brereton G. éd (2018), The BP exhibition: I am Ashurbanipal king of the World, King of Assyria, Londres.

Casanova M. (2008), « Vases à parfums et boîtes à fard du Proche-Orient ancien, de l'Asie centrale à la Méditerranée orientale, $4^{\mathrm{e}}-2^{\mathrm{e}}$ millénaires av. J.-C. ", dans Bodiou L., Frère D. et Mehl V. éd, Parfums et odeurs dans l'Antiquité, Rennes, p. 167-178.

Charpin D. (2017), La vie méconnue des temples mésopotamiens, Docet omnia 1, Paris.

Cousin L. (2016), « Beauty Experts: Female Perfume Makers in the 1st millennium BC », dans Lion B. et Michel C. éd., The Role of Women in Work and Society in the Ancient Near East, SANER 13, Berlin.

Cousin L. (à paraître), « The Scents of Babylonian Sanctuaries ", dans Cousin L., Quillien L. et Ramez M. éd, Material Culture of Babylonia and Beyond, OLA, Paris-Louvain-Walpole.

Da Riva R. et Galetti G. (2018), « Two Temple Rituals from Babylon », Journal of Cuneiform Studies 70, p. 189-227.

Durand J.-M. (2009), La nomenclature des habits et des textiles dans les textes de Mari. Matériaux pour le Dictionnaire de Babylonien de Paris. Tome 1, Archives Royales de Mari 30, Paris.

Fales F. M. (2004), « Rileggendo gli inventari di Qatna », Kaskal : rivista di storia, ambiente e culture del Vicino Oriente Antico 1, p. 83-127.

Farber W. (1987), « Tamarisken-Fibeln-Skolopender », dans Rochberg-Halton F. éd., Language, Literature, and History: Philological and Historical Studies Presented to Erica Reiner, American Oriental Series 67, New Haven, p. 85-105.

Farber W. (2014), Lamaštu: An Edition of the Canonical Series of Lamaštu Incantations and Rituals and Related Texts from the Second and First Millennia B.C., Mesopotamian Civilizations 17, Winona Lake.

Frontisi-Ducroux F. et Vernant J.-P. (1997), Dans l'œil du miroir, Paris.

Goddeeris A. (2016), The Old Babylonian Legal and Administrative Texts in the Hilprecht Collection Jena, Texte und Materialen der Hilprecht Collection 10, Wiesbaden.

Guichard M. (2005), La vaisselle de luxe des rois de Mari. Matériaux pour le Dictionnaire de Babylonien de Paris. Tome II, Archives Royales de Mari 31, Paris.

Gurney O. R. (1953), « Further Texts from Dur-Kurigalzu », SUMER 9, 1953, p. 21-34. 
Hoffner H. A. (1966), « Symbols for Masculinity and Feminity: Their Use in Ancient Near Eastern Sympathetic Magic Rituals », Journal of Biblical Literature 85, p. 326-334.

Hussein M. M. (2016), Nimrud: The Queen's Tombs, Bagdad-Chicago.

Husson C. (1977), L'offrande du miroir dans les temples égyptiens de l'époque gréco-romaine, Lyon. Jacquet A. (à paraître), Texts from Lagaba, Babylonian Inscriptions in the Collection of James B. Nies 11, New Haven-Londres.

Joannès F. (1992), «Les temples de Sippar et leurs trésors à l'époque néo-babylonienne », Revue d'Assyriologie et d'archéologie orientale 86, p. 159-184.

Joannès F. (2005), « L'argent des dieux babyloniens au VI s. av. J.-C. », Topoi 12-13, p. 35-54.

Joannès F. (2006), « Traitement des malades et bit hilși en Babylonie », dans Battini L. et Villard P. éd., Médecine et médecins au Proche-Orient ancien, British Archaeological Reports 1528, Oxford, p. 73-90.

Jursa M. (2007), « Die Söhne Kudurrus und die Herkunft der neubabylonischen Dynastie », Revue d'Assyriologie et d'archéologie orientale 101, p. 125-136.

Kramer S. N. (1967), « The Death of Ur-Nammu and His Descent to the Netherworld », Journal of Cuneiform Studies 21, p. 104-122.

Leemans W. F. (1952), Ishtar of Lagaba and her dress, Studia ad tabulas cuneiformes collectas a F.M. Th. de Liagre Bohl pertinentia 1-1, Leyde.

Maggio M. (2012), L'ornementation des dieux à l'époque paléo-babylonienne, Alter Orient und Altes Testament 393, Münster.

Middeke-Conlin R. (2014), « The Scents of Larsa: A Study of the Aromatics Industry in an Old Babylonian Kingdom », Cuneiform Digital Library Journal 1 [en ligne], https://cdli.ucla.edu/pubs/ cdlj/2014/cdlj2014_001.html

Moore E. W. (1975), Neo-Babylonian Business and Administrative Documents, Ann Arbor.

Moran W. L. (1987), Les lettres d'El-Amarna. Correspondance diplomatique du pharaon, Littératures Anciennes du Proche-Orient 13, Paris.

Muller V. (2015), « Les tablettes cunéiformes des premières fouilles de Tell Mishrifeh/Qațna : état de la question ", dans Pfälzner P. et Al-Maqdissi M. éd., Qatna and the Networks of Bronze Age Globalism. Proceedings of an International Conference in Stuttgart and Tübingen in October 2009, Qatna Studien Supplementa 2, Wiesbaden, p. 223-228.

Nemet-Nejat K. Rh. (1993), « A Mirror Belonging to the Lady-of-Uruk », dans Cohen M. E., Snell D. C. et Weisberg D. B. éd., The Tablet and The Scroll: Near Eastern Studies in Honor of William W. Hallo, Bethesda, p. 163-169.

Ornan T. (2002), «The Queen in Public: Royal Women in Neo-Assyrian Art », dans Parpola S. et Whiting R. M. éd., Sex and Gender in the Ancient Near East: Proceedings of the $47^{\text {th }}$ Rencontre Assyriologique Internationale, Helsinki, July 2-6, 2001, vol. II, Helsinki, p. 461-477.

Potts D. T. et al. (1996), « GuHllu and Guggulu », Wiener Zeitschrift für die Kunde des Morgenlandes 86, p. 291-305.

Prieur A. (2005), « Les coquillages du Paléolithique à l'âge du Bronze au Moyen-Orient et en Méditerranée orientale : interprétations environnementales et utilisation humaine ", Paléorient 31-1, p. 158-168. 
Rova E. (2008), « Mirror, Distaff, Pomegranate, and Poppy Capsule: on the Ambiguity of Some Attributes to Women and Goddesses ", dans Kühne H., Czichon R. M. et Kreppner F. J. éd., 4 ICAANE. Proceedings of the 4th International Congress on the Archaeology of the Ancient Near East, vol. I, Wiesbaden, p. 557-570.

Schaudig H. (2001), Die Inschriften Nabonids von Babylon und Kyros' des Großen samt den in ihrem Umfeld entstandenen Tendenzschriften, Alter Orient und Altes Testament 256, Münster.

Simpson S. J. (2018), « Small, Bright and Colorful: Observations on the Circulation of Minor Glass objects from Sassanian Contexts ", dans de Vigo P. éd, Le archeologie di marilli: Miscellanea di studi in ricordo di Maria Maddalena Negro Ponzi Mancini, Alexandrie, p. 105-120.

Steinkeller P. (2012), « New Light on Marhaši and its Contacts with Makkan and Babylonia », dans Giraud J. et Gernez G. éd., Aux marges de l'archéologie. Hommage à Serge Cleuziou, Travaux de la Maison René-Ginouvès 16, Paris, p. 261-274.

Svärd S. (2015), Women and Power in Neo-Assyrian Palaces, State Archives of Assyria Studies 23, Helsinki.

Westenholz J. G. (2010), « Drink me only with thine eyes », dans Melville S. C. et Slotsky A. éd., Opening the Tablet Box: Near Eastern Studies in Honor of Benjamin R. Foster, Culture and History of the Ancient Near East 42, Leyde-Boston, p. 463-484.

\section{NOTES}

1. Voir collections.louvre.fr/ark:/53355/cl010120474

2. Voir etcsl.orinst.ox.ac.uk, texte Dumuzid-Inana E1 : c.4.08.31.

3. Voir etcsl.orinst.ox.ac.uk, texte The death of Ur-Namma, Ur-Namma A: c.2.4.1.1.

4. Concernant le lien entre le peigne, la quenouille et l'huile, on peut également renvoyer à l'étude de Françoise Frontisi-Ducroux et Jean-Pierre Vernant (1997, chapitre 3), qui ont montré que ces deux objets peuvent cohabiter dans les représentations iconographiques de la Grèce ancienne, étant donné qu'ils font partie des intérieurs athéniens.

5. www.penn.museum/collections/object/121898

\section{RÉSUMÉS}

En Mésopotamie, les statues sont considérées comme de véritables incarnations de la divinité, qui doivent être nourries et habillées. Leurs temples abritent par conséquent de véritables trésors, des accumulations de richesses sous la forme d'objets de culte (vaisselle sacrée en or ou en argent), ou de bijoux dont les officiants parent les statues. Fondée sur les données textuelles en écriture cunéiforme et sur certains objets découverts grâce aux fouilles archéologiques au Proche-Orient, cette contribution cherche à déterminer, d'une part, si les miroirs, les peignes et les récipients à cosmétique, mentionnés dans la documentation de la pratique des temples, font 
partie de la vaisselle et de la joaillerie exclusivement attribuées aux déesses, et, d'autre part, dans quelle mesure ils sont associés plus largement à la féminité dans la société mésopotamienne.

In Mesopotamia, statues are considered actual incarnations of the divinity, which must be sustained and clothed. Their temples therefore house real treasures, accumulations of wealth in the form of cult objects (sacred vessels made of silver or gold) or jewellery for the officiants to use for adorning the statues. Based on textual data in cuneiform writing and on certain objects discovered through archaeological excavations in the Near East, this contribution attempts to determine, on the one hand, whether mirrors, combs and cosmetic containers, mentioned in the administrative documentation of the temples, are part of the vessels and jewellery exclusively attributed to the goddesses and, on the other hand, to what extent they are more broadly associated with femininity in Mesopotamian society.

INDEX

Mots-clés : miroir, peigne, cosmétique, récipient à cosmétique, déesse, sanctuaire

Keywords : mirror, comb, cosmetics, cosmetic container, goddess, sanctuary

\section{AUTEUR}

LAURA COUSIN

ArScAn UMR 7041, équipe Histoire et archéologie de l'Orient cunéiforme 